\title{
Stimulus discriminability in the magnitude estimation and category rating of loudness
}

\author{
I. D. JOHN 1 \\ UNIVERSITY OF ADELAIDE
}

Thirty-three $S s$ made category ratings and magnitude estimations of 10 auditory stimuli differing in loudness. The results from each task were examined in terms of the response uncertainty conditional upon each stimulus. The results did not support the suggestion that category judgments are influenced by the relative discriminability of stimuli in a way which is not characteristic of magnitude estimates, but were found to be consistent with the subjective standard hypothesis. It is argued that the observed quasi-logarithmic relationship between category scale and ratio scale values reflects the constraints placed upon responses in category rating tasks.

The differences between the properties of ratio scales and category scales have continued to command wide interest since they were first discussed by Stevens and Galanter (1957). In the case of Class 1 or prothetic stimulus continua, category scales are concave downward when plotted against ratio scales. The form of the relationship may perhaps best be described as quasi-logarithmic since it appears to be intermediate between a linear and true logarithmic relationship.

It has been suggested (Stevens, 1957) that "The chief factor that produces nonlinearity in the category scales of Class 1 is variation in the S's sensitivity to differences. Near the lower end of the scale where discrimination is good, the categories tend to be narrow, and by consequence the slope of the function is steep. Near the upper end, where a given stimulus difference is less easy to detect, the categories broaden and the slope declines."

Although some doubts have been cast on this explanation and a number of alternative explanations of the observed relationship have been advanced, it does not seem to have been subjected to direct empirical test. Torgerson (1960), for example, has suggested that while ratio scales may describe the subjective ratios between stimulus magnitudes, category scales describe the subjective differences between stimulus magnitudes. It can be deduced from this position that a category scale of darkness will be the reverse of a category scale of lightness, that a ratio scale of darkness will be the reciprocal of a ratio scale of brightness and that the relationship between category and ratio scales will be logarithmic in each case. Torgerson's experimental evidence is, on the whole, consistent with these predictions although minor departures were shown to exist from the postulated logarithmic relationship between category and ratio scales.

Eisler (1962a, b) has suggested that a precise logarithmic relationship may obtain between category scale values and ratio scale values if the ratio scale values are corrected by the addition of a parameter $\mathrm{q} / \mathrm{k}$, which is derived from the variance of the ratio scale values. Experimental evidence consistent with this suggestion has been obtained but the psychological significance of the parameter $q / k$ has not been satisfactorily clarified.

A third explanation has been offered by Treisman (1964), who has argued that the most probable form of the relationship between stimulus intensity (I) and the magnitude of the central neural effect of the stimulus is logarithmic. The $S$, according to this view, is usually able to carry out the relatively simple exponential transformation between stimulus and response $(R)$, which is required to produce a ratio scale. It is postulated that the precise form of the exponential transformation which the $S$ employs is learned on the basis of past experience (Warren, 1958). It is further postulated that the requirement of category scaling, to select equal intervals on the scale of $I$ is mathematically so complex that Ss use a linear transformation between stimulus and $\mathrm{R}$ or compromise between a linear and exponential transformation.

Recent evidence concerning the manner in which the properties of both ratio scales (Poulton, 1968) and category scales (Parducci, 1963) may be quite markedly influenced by incidental experimental manipulations suggests that the search for a precise specification of the relationship between these two kinds of scales is perhaps premature until such time as "error free" scales of both kinds can be produced.

The study which is to be described was not primarily concerned with clarifying the precise relationship between category and ratio scales, but had as its aim a direct examination of Stevens' assertion that category judgments are influenced by the relative discriminability of stimuli in a way which is not characteristic of magnitude estimates. The reciprocal of response uncertainty conditional upon the particular stimulus $\left[\mathrm{Us}_{\mathrm{i}}(\mathrm{R})\right]$, a measure derived from information analysis, was used as a comparative measure of stimulus discriminability.

The value of $\mathrm{Us}_{\mathrm{i}}(\mathrm{R})$ for each stimulus $\left(\mathrm{s}_{\mathrm{i}}\right)$ may be calculated by means of the formula:

$$
\mathrm{Us}_{\mathrm{i}}(\mathrm{R})=-\sum_{\mathbf{j}} \mathrm{p}_{\mathrm{j}} \log _{2} \mathrm{p}_{\mathrm{j}}
$$

where $p_{j}$ is the proportion of occasions that the stimulus is associated with response $\mathrm{j}$ and the summation runs over all alternative responses. The maximum possible value of $\mathrm{Us}_{\mathrm{i}}(\mathrm{R})$ will be determined by the experimental constraints placed upon the $S$; thus, in a category rating task it will be $\log _{2}$ of the number of categories and in a magnitude estimation task it will be $\log _{2}$ of the number of repetitions of the stimulus. Although $\mathrm{Us}_{\mathrm{i}}(\mathrm{R})$ values from different experimental conditions are therefore not directly comparable, comparisons between $\mathrm{Us}_{\mathrm{i}}(\mathrm{R})$ values of the stimuli under the same experimental conditions are quite meaningful.

Two additional properties of this measure are also of some importance; its use involves no assumptions concerning the properties of the metric underlying either the stimulus or response systems. Secondly as it may be influenced by both stimulus and response factors it cannot be regarded as the basis of a completely unambiguous measure of stimulus discriminability. However, since changes in stimulus discriminability must produce changes in $U_{S_{i}}(R)$ except in the unlikely case that they are exactly compensated for by changes in response discriminability, the use of $\mathrm{Us}_{\mathrm{i}}(\mathrm{R})$ as a comparative measure of stimulus discriminabilitv seems iustified.

\section{Subjects}

\section{METHOD}

Thirty-three members of a second year class in psychology at the University of Adelaide served as $\mathrm{Ss}$ in the main experiment. The Ss had no previous experience of experiments of this kind and completed the magnitude estimation (ME) task and category rating (CR) task in two sessions one week apart. Two additional Ss completed the ME task only and a further 40 comparable Ss took part in an additional ME task. 


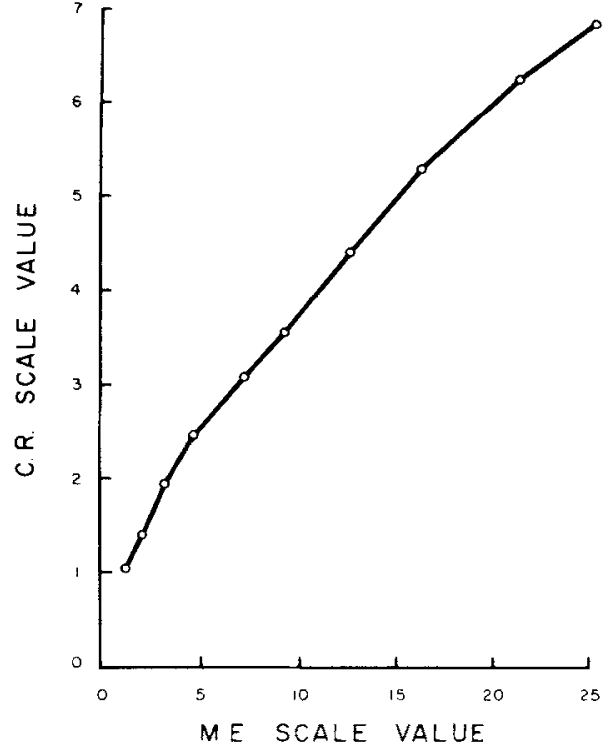

Fig. 1. Relationship between magnitude estimates and category ratings of loudness.

\section{Stimuli}

The task was group administered in a large laboratory with the stimuli presented through a loudspeaker. The stimuli consisted of 101000 -cps tones separated by steps of $5 \mathrm{~dB}$ and produced by an audio signal generator. The least intense stimulus was cleariy audible to all Ss; the duration of each stimulus was $2 \mathrm{sec}$. The individual stimuli were presented in 11 blocks of 10 stimuli, with each stimulus appearing once in each randomly ordered block of 10 . The responses from only the last 100 stimulus presentations were utilized for the major analysis.

\section{Instructions}

Under the MF condition. Ss were given the conventional magnitude estimation instructions (Stevens, 1961) with the request that the loudness of the fifth loudest stimulus in the sertes $(20 \mathrm{~dB})$, which was demonstrated twice, be called " 10 ." Under the CR condition, the loudest and softest stimuli were demonstrated and Ss were requested to assign all stimuli to one of seven categories, using " $l$ " for the softest sound and " 7 " for the loudest.

\section{RESULTS}

Scale values were determined for the ME condition by utilizing the mean of Ss' median responses to each stimulus (Stevens, 1961) and for the CR condition by utilizing the mean of Ss' mean responses. The CR scale values when plotted against the MF scale values produced the expected type of function, that is, concave downwards. When CR scale values were plotted against log-ME scale values the function was not linear and hence the relationship between the two scales, in this case, is quast-logarithmic.

The values of $U s_{j}(R)$ associated with each of the 10 stimali employed for both $\mathrm{CR}$ and $\mathrm{ME}$ conditions were calculated for each $\mathrm{S}$.

The $U_{s}(R)$ values were examined by analysis of variance. As anticipated experimental conditions proved to be a highly significant source of variance $(F=1,120$, df $=1 / 288$, $\mathrm{p}<.001$ ); the interaction of individual stimulus values and experimental conditions was, however, of only marginal significance $(F=226, \mathrm{df}=9 / 288, .01<\mathrm{p}<.05)$.

The relationship between $U_{s}(R)$ and stimulus intensity
(Fig. 2) may be seen to be very similar for both experimental conditions. Those stimuli nearest the ends of the stimulus range are most discriminable and those nearest the center of the stimulus range are least discriminable.

Under the $\mathrm{ME}$ conditions the values of $U \mathrm{~s}_{\mathrm{i}}(\mathrm{R})$ associated with the standard stimulus $(20 \mathrm{~dB})$ is less than that associated with the two adjacent stimuli. This finding appears to reflect the greater response strength of the response " 10 ," which was used on a mean number of 7.6 occasions by each $\mathrm{S}$; the mean number of occasions on which each response other than " 10 " was used by each $\mathrm{S}$ was 3.8 .

In order to investigate the possibility that the ME results may have been determined by the particular response conditions imposed on Ss, an additional 40 Ss completed the ME task under exactly the same conditions with the exception that they were told in the initial instructions that the loudness of the standard stimulus $(20 \mathrm{~dB})$ was to be called " 100 ." The relationship between $U_{s_{i}}(R)$ and stimulus intensity for this group was essentially the same.

Since in most magnitude estimation experiments the individual stimuli are seldom repeated as often as 11 times, the possibility that the obtained functions resulted from prolonged experimentation was examined. The responses of all Ss completing the $\mathrm{MF}$ task $(\mathrm{N}=75)$ were examined to determine the number of Ss making the same response on the first two presentations of each stimulus. These results (Table 1) indicate that the obtained pattern is established very early in the course of the experiment.

\section{DISCUSSION}

These results provide no support for Stevens' view that the effects of stimulus discriminability operate in a different fashion in $C R$ and ME tasks but suggest that there is no essential difference in the operation of this variable in either task.

On this evidence alone it is not possible to state precisely how Ss do make judgments in tasks of this kind, but it may be observed that the findings are consistent with Eriksen and Hake's $\left(195^{7}\right)$ subjective standard hypothesis. which states that "a $S$. when confronted with a series of stimuli in an dbsolute judgment task. selects certam stimuli that he uses as standards for judging the remaining stmmuli. When a stimulus is presented, the $S$ attempts to recall one of the standard stimuli and uses it as a reference in judging the presented stimulus." In this case the subjective standards appear to be the extreme stimuli of the stimulus range and the values of $\mathrm{Us}_{\mathbf{i}}(\mathrm{R})$

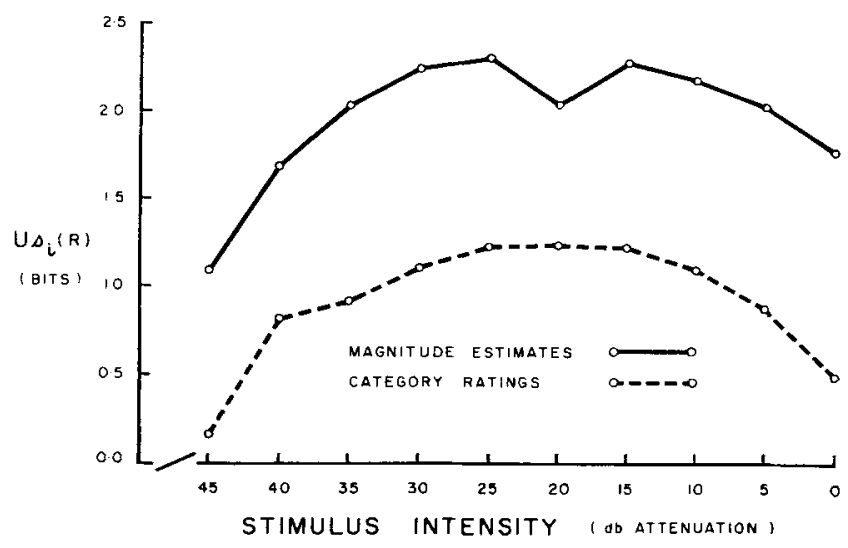

Fig. 2. Relationship between response uncertainty conditional upon individual stimulus $\left[U s_{i}(R)\right]$ and stimulus value for magnitude estimates and category ratings of loudness. 
Table 1

Number of Ss Giving the Same Response on the First Two Presentations of Each Stimulus in the Magnitude Estimation Task $(N=75)$

Stimulus Intensity (dB attenuation)

\begin{tabular}{rrrrrrrrrrr} 
& 45 & 40 & 35 & 30 & 25 & 20 & 15 & 10 & 5 & 0 \\
No. of Ss & 37 & 13 & 10 & 2 & 19 & 10 & 8 & 8 & 12 & 26 \\
\hline
\end{tabular}

associated with the remaining stimuli increase as a function of their distance from the nearest subjective standard.

If the validity of the subjective standard hypothesis is tentatively accepted it is possible to advance an alternative explanation to account for the observed quasi-logarithmic relation between ratio and category scales. Since in judgment situations of this kind Ss appear to utilize two standards (i.e., the extreme stimulus values), it would seem that scales based on data of this kind are not unitary, but are functionally two partially independent semiscales. In the ME task $\mathrm{S}$ is initially given a value for a stimulus in the middle of the stimulus range, and is required to determine response values for the two subjective standards. It would appear that these values are chosen, probably on the basis of S's knowledge of the appropriate physical correlate (Warren, 1958), so that the properties of the two semiscales are consistent. In the CR task, however, the response values of the two subjective standards are assigned by $\mathrm{E}$, and $\mathrm{S}$ is required to divide up the available responses between the two semiscales.

It seems that this is accomplished by allocating an equal number of scale units to each of the semiscales. The point at which the two semiscales join coincides with that stimulus value about which there is greatest response uncertainty. Because of the relativity of judgment the greatest response uncertainty is not associated with a stimulus value in the precise center of the range but is usually associated with a stimulus value near the geometric mean of the stimulus range. Helson (1964) has shown that for a large number of stimulus continua the mean CR value usually corresponds closely to the geometric mean of the stimulus range.

If the CR scale values of three points on the stimulus continuum are determined in this fashion the properties of the $C R$ scale are largely fixed and $S$ is required only to interpolate $C R$ values for the remaining stimuli in a consistent fashion. It follows from this argument that $C R$ values should approximate a linear function of the log of the stimulus values and hence be approximately linearly related to log-ME values. A slight departure from this relationship is likely to arise from the end stimulus effect and truncation of the response scale, with the consequence that the scale values of those stimuli near the ends of the stimulus range will be somewhat displaced towards the middle of the scale.

This explanation of the relationship between category scales and ratio scales is also consistent with the linear relationship between the two types of scales obtained with the stimulus continuum of pitch which is the apparent ideal case of a Class 2 or metathetic stimulus continuum. Over a very wide range (about $60 \mathrm{cps}-1000 \mathrm{cps}$ ) the difference limen for pitch is roughly constant at about $2.3 \mathrm{cps}$; even above this range the value of the Weber fraction is small by comparison with most other stimulus continua (Licklider, 1951). Both of these factors should operate to produce a linear relationship between ratio and category scales, the first by tending to equalize the stimulus range covered by the two semiscales, and the second by reducing effects due to truncation of the response scale.

\section{REFERENCES}

EISLER, H. On the problem of category scales in psychophysics. Scandinavian Journal of Psy chology, 1962a, 3, 81-87.

EISLER, H. Empirical test of a model relating magnitude and category scales. Scandinavian Journal of Psychology, 1962b, 3, 88-96.

ERIKSEN, C. W., \& HAKE, H. W. Anchor effects in absolute judgments. Journal of Experimental Psychology, 1957, 53, 132-138.

HELSON, H. Adaptation-level theory. New York. Harper \& Row, 1964

LICKLIDER, J. C. R. Basic correlates of the auditory stimulus. In S. S. Stevens (Ed.), Handbook of experimental psychology. New York. Wiley, 1951.

PARDUCCI, A. Range-frequency compromise in judgment. Psychological Monograph, 1963, 77, No. 565.

POULTON, E. C. The new psychophysics: Six models for magnitude estimation. Psychological Bulletin, 1968, 69, 1-19.

STEVENS, S. S. On the psychophysical law. Psychological Review, 1957, $64,153-181$

STEVENS, S. S. The psychophysics of sensory function. In W. A. Rosenblith (Ed.), Sensory communication. Cambridge, Mass.: M.I.T. Press, 1961.

STEVENS, S. S., \& GALANTER, E. W. Ratio scales and category scales for a dozen perceptual continua. Journal of Experimental Psychology, $1957,54,377-411$.

TREISMAN, M. Sensory scaling and the psychophysical law. Quarterly Journal of Experimental Psychology, 1964, 16, 11-22.

TORGERSON, W. S. Quantitative judgment scales. In H. Gulliksen and S. Messick (Eds.), Psychological scaling. Theory and applications. New York: Wiley, 1960.

WARREN, R. M. A basis for judgments of sensory intensity. American Journal of Psychology, 1958, 71, 675-687.

NOTE

1. Address: Department of Psychology, University of Adelaide, Adelaide, South Australia 5001.

(Accepted for publication November 27, 1968.) 